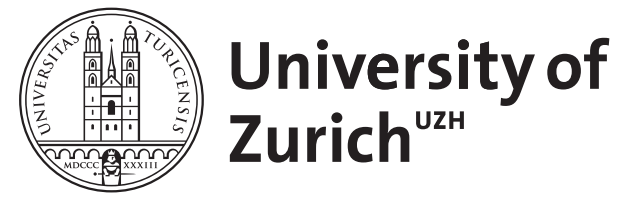

\title{
Vedische exozentrische Komposita mit drei Relationen
}

Scarlata, Salvatore ; Widmer, Paul

DOI: https://doi.org/10.1163/15728536-0580010

Posted at the Zurich Open Repository and Archive, University of Zurich ZORA URL: https://doi.org/10.5167/uzh-104846

Journal Article

Accepted Version

Originally published at:

Scarlata, Salvatore; Widmer, Paul (2015). Vedische exozentrische Komposita mit drei Relationen. IndoIranian Journal, 58(1):26-47.

DOI: https://doi.org/10.1163/15728536-0580010 


\title{
Vedische exozentrische Komposita mit drei Relationen
}

\author{
Salvatore Scarlata und Paul Widmer \\ Universität Zürich \\ Indogermanistik, Rämistr. 68, CH-8oo1 Zürich, paul.widmer@uzh.ch
}

\begin{abstract}
In this paper it is suggested that a large subset of Vedic exocentric nominal compounds can best be described by focusing on two semantosyntactic relationships that exist in addition to the first one, viz. the modificative relation an exocentric compound bears to the head noun: A second, external relation which establishes a link between one single member of the compound and the noun the compound modifies as a whole, and a third, internal relation which combines the two members of a compound to form a semantosyntactic unit. For both, internal and external relations, a distinct set of three preferred readings is established which provides a semantosyntactic framework for interpreting these compounds. It is emphasized that a correct interpretation always depends not only on these sets of possible readings, but, most importantly, on the semantics of the compound members and the modified noun as well as on the pragmatics and the context the syntagm occurs in.
\end{abstract}

Keywords: Vedic language, possessive compounds, verbal governing compounds, causative compounds, semantic relations.

aop; accepted for publication in Indo-Iranian Journal 


\section{Einleitung}

Eine allgemeingültige Definition und Kategorisierung von Komposition und Komposita ist notorisch schwierigi und aus sprachübergreifender Perspektive lassen sich bisher nur beschränkt Generalisierungen formulieren. ${ }^{2}$ Bestimmt wird die jeweilige Kategorisierung insbesondere auch durch die gewählte Perspektive: Eine diachrone Definition unterscheidet sich zwangsläufig von einer kognitiven Definition, eine syntaktische von einer morphologischen etc. Zudem kann eine Definition, die z. B. für das Deutsche einschlägig ist, in einer anderen Sprache an der Unvergleichbarkeit der beiden Sprachsysteme und den gänzlich verschiedenen sprachinternen und sprachexternen Vorausetzungen und Klassifikationskriterien scheitern. Die Beschreibung einer Klasse rigvedischer Komposita in diesem Beitrag ist deshalb bewusst in dem Sinn nicht einer Theorie verpflichtet, dass nicht versucht wird, in einem top-down-Verfahren ein bestimmtes Beschreibungsmodell zu verwenden oder die Anwendbarkeit eines Beschreibungsmodells am Rigveda zu überprüfen. Die Herangehensweise ist dabei vorerst synchron gehalten und konzentriert sich auf die Identifizierung der einzelnen Typen und nicht auf die Erfassung aller Einzelbelege. Der Fokus auf die Synchronie überdeckt den Umstand, dass wir einzelnen Lexemen nicht immer gerecht werden können, wenn sie z. B. auf eine komplexe individuelle oder strukturelle Vorgeschichte zurückblicken. Die grosse Menge der synchron bildbaren und tatsächlich gebildeten Komposita des betreffenden Typs, die eine solide Basis für weitere Überlegungen bildet, ist damit aber repräsentiert.

Die Beschreibung und Kategorisierung der rigvedischen exozentrischen Komposita blickt auf eine lange Tradition zurück, auf der die hier vorgenommene relationale Einteilung naturgemäss aufbaut. Zu den wichtigsten Beiträgen gehören neben der grundlegenden Darstellung von Wackernagel (1905) unter anderen Justi (1861), Whitney (1896), Jacobi (1897), Brugmann (1906), Macdonell (1910), Schindler (1986), Schindler (1997), Scarlata (1999), Sadovski (2000), Sadovski (2004), Lühr (2004) und Kastovsky (2009). ${ }^{3}$

Es wird hier ein Zugang gewählt, der weniger morphologische Operationen, die Bestimmung verwendeter Wortklassen oder den Akzentsitz in den Vordergrund rückt, sondern funktional das Spektrum der realisierten semantisch-syntaktischen Relationen beschreibt, die zwischen dem Kompositionsvorderglied, dem Kompositionshinterglied und dem modifizierten Begriff bestehen können. Diese Herangehensweise ermöglicht es, im Sinn von Lühr (2004) die synchronen Interpretationsstrategien und -optionen zu identifizieren, die durch die gängige Neutralisierung von Genus- und Numerusunterschieden und durch das verbreitete Unterbleiben morphosyntaktischer Auszeichnung der Relationen notwendig werden (Schindler 1997; Lühr 2004, 86-94).

1 S. Lieber \& Štekauer (2009); Scalise \& Bisetto (2009), mit verschiedenen Systematiken und Ansätzen auch aus älterer Literatur auf S. 39-41.

2 S. Bauer 2009.

3 Weitere Literatur bei Lindner (2011-). 
Im gleich anschliessenden Kapitel wird zuerst erläutert, mit welchen drei grundlegenden Relationen in vedischen Syntagmen bestehend aus einem Bezugswort und einem exozentrischen Kompositum wir operieren. Darauf folgt die Beschreibung der semantisch-syntaktischen Eigenschaften der Relationen, die hier extern (S. 4ff.) und intern (S. 8ff.) genannt werden. ${ }^{4}$

\section{Trirelationalität exozentrischer Komposita}

Behandelt werden im Folgenden vedische exozentrische Komposita, i. e. aus zwei Lexemen X und Y zusammengesetzte Lexeme, die eine akzentuelle Einheit bilden und exozentrisch in dem Sinne sind, dass das Gesamtkompositum keine Instanz von einem der beiden Kompositionsglieder bezeichnet wie in Bsp. (1).

(1) $[\operatorname{tigm}(\dot{a})-\bar{a} y u d h a-]$ índra-

[ scharf Waffe ] Indra

,Indra mit seiner scharfen Waffe

Das Besondere an einem Syntagma aus einem attributiv verwendeten Kompositum und einem Bezugswort wie in (1) besteht darin, dass systematisch drei Relationen identifizierbar sind. ${ }^{5}$ Eine erste Relation besteht zwischen dem Gesamtkompositum (in Bsp. (1): tigmáy yudha-) und dem durch das attributiv verwendete Kompositum modifizierten Substantiv (in Bsp. (1): índra-).

Eine zweite Relation besteht zwischen einem einzelnen Glied des Kompositums und dem modifizierten Begriff. In (1) betrifft dies das Bezugsnomen índra- und das Kompositionshinterglied -āyudha-. Dem Bezugsnomen índra- kommt dabei die Funktion zu, die Waffe (áyudha-) näher zu spezifizieren. Bei dieser zweiten Relation handelt es sich also semantisch um eine Subklassifizierung, in der die Waffe als die des Indra bestimmt wird. Das semantische Verhältnis zwischen índra- und -āyudha-ist somit ein Modifikationsverhältnis, das in diesem Fall morphosyntaktisch nicht eigens bezeichnet wird; es existiert allein aufgrund der Tatsache, dass índra- durch das Gesamtkompositum modifiziert wird.

Durch diese zweite Relation entsteht die Situation, dass das Kompositionshinterglied -āyudha- als Teil des Gesamtkompositums einerseits zur Modifizierung des syntaktischen Kopfs índra- beiträgt, wobei die Modifikation durch die Kongruenz zwischen dem Gesamtkompositum und dem Bezugsnomen morphosyntaktisch ausgedrückt wird. Gleichzeitig modifiziert der Kopf der Phrase (índra-) das Kompositionshinterglied (-āyudha-). Der durch das Gesamtkompositum modifizierte Begriff modifiziert also seinerseits gleichzeitig einen Bestandteil des Kompositums. ${ }^{6}$

4 Eindeutig substantivierte Komposita wie z. B. Eigennamen werden hier nicht berücksichtigt. Funktional verfügen Komposita insofern über ein grosses Potential, als sie als komplexe Satzäquivalente verwendet werden können. Darauf wird an dieser Stelle nicht weiter eingegangen, doch vgl. die Übersetzungen zu den zitierten Stellen, z. B. in Fn. 28.

5 In einigen Fällen sind weitere Relationen vorhanden, die aber nicht systematisch auftreten; s. auch Bsp. (13a).

6 Zur Funktion der Modifikation s. u. S. 4f. (Possessive Modifikation). Zu anderen Relationen s. S. 5 ff. (Kausativ) und S. 7 f. (S/A-Verb-Relation). 
Die dritte Relation besteht in (1) zwischen dem Kompositionsvorderglied tigm(á)- und dem Kompositionshinterglied -āaydha-. Die Relation kann wahlweise prädikativ oder als attributive Modifikation aufgefasst werden.

Es bestehen also drei distinkte Relationen, die ganz allgemein folgendermassen dargestellt werden können:

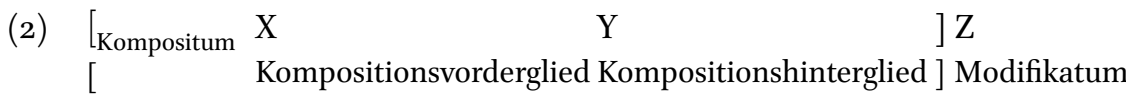

1. Gesamtkompositum $\leftrightarrow \mathrm{Z}$

2. Externe Relation: $\mathrm{Z} \leftrightarrow \mathrm{Y}(/ \mathrm{X})^{7}$

3. Interne Relation: $\mathrm{X} \leftrightarrow \mathrm{Y}$

\section{Semantische und syntaktische Eigenschaften der externen und internen Relationen}

Wesentlich für die hier behandelten Komposita sind die Eigenschaften der externen und internen Relationen, die die Interpretationsmöglichkeiten auffächern und auch einschränken und auf deren Hintergrund die Komposita bzw. die Syntagmen, in denen sie auftreten, ihre Bedeutungen entfalten. Im folgenden wird die Beschreibung der semantisch-syntaktischen Eigenschaften dieser Relationen vorgenommen.

\section{Externe Relation}

In der externen Relation lassen sich drei grundlegende Typen mit verschiedenen Eigenschaften identifizieren.

\section{Possessive Modifikation}

Im ersten Typ modifiziert Z ein Kompositionsglied (meist Y).

(3) $[\operatorname{tigm}(a ́)-\bar{a} y u d h a-]$ índra-

[ scharf Waffe ] Indra

$\left[\begin{array}{lll}\mathrm{X} & \mathrm{Y}\end{array}\right] \mathrm{Z}$

,Indras Waffe ... ${ }^{8}$

7 Im Vergleich zu āsánnișu- ,mit Pfeilen im Munde‘ scheint in ișuhasta- ,mit Pfeilen in der Hand’ eine Relation zwischen Z und $\mathrm{X}$ zu bestehen. In einigen Fällen ist dies durchaus möglich, relevant ist hier lediglich die Existenz einer spezifischen Relation zwischen Z und einem Kompositionsglied. S. hierzu weiter unten zu S. 8f. mit den Beispielen (12a) und (12b).

8 2.30.3d: tigmáyudho ajayac chátrum índrah ,Mit seiner scharfen Waffe besiegte Indra den Gegner'; vgl. noch 5.2.10, 6.74.4, 7.46.1, 9.90.3. 
(4) [ghrtá-yoni- ] agní-

[ Butter Stätte ] Agni

$\left[\begin{array}{lll}\mathrm{X} & \mathrm{Y}\end{array}\right] \mathrm{Z}$

,Agnis Stätte ... ${ }^{9}$

In Beispiel (3) modifiziert Z (índra) das Kompositionshinterglied Y (-āyudha-,Waffe'), indem es das Kompositionshinterglied subklassifiziert. Ebenso in (4), wo -yoni- Stätte` (Y) als die des Agni (Z) spezifiziert wird.

So wie auch sonst ein Modifikationsverhältnis zwischen zwei Substantiven typischerweise eine possessive Funktion übernimmt, wird auch in diesem Fall eine Possessivrelation etabliert mit $\mathrm{Z}$ als Possessor und Y als Possessum, wodurch die Interpretation dieses Typs als Possessivkompositum zustande kommt. Dieses Possessivverhältnis wird innerhalb des Syntagmas aus Kopf und Kompositum nicht explizit ausgedrückt. In weniger kondensierten Konstruktionen mit expliziter Auszeichnung der Verhältnisse kann diese Relation morphosyntaktisch z. B. mit dem Genitiv ausgedrückt werden. Ein nicht-komponiertes Korrelat für Bsp. (3) findet sich in RV 10.108.5d: asmákam áyudhā santi tigmá ,unsere (Gen.) Waffen sind scharf' bzw. für Bsp. (4) in RV 2.3.11a ghrtám asya yónis ,Schmalz (ist) dessen Stätte‘.

\section{Kausativ}

Im zweiten Typ besteht zwischen Y und Z eine kausativische Relation (s. zur Semantik bereits Schroeder 1874, 199f., 379; Schindler 1986, 398, besonders Fn. 17; Lühr 2004, 55), wobei die Kausativität weder morphosyntaktisch noch lexikalisch ausgedrückt wird. Z fungiert dabei als Verursacher (causer) in der A-Funktion ${ }^{10}$ der internen Prädikation und Y hat die P-Funktion des causee inne wie in Bsp. (5).

(5) [vrșțí- dyu- ]páti-

[ Regen Himmel ] Herr

$\left[\begin{array}{lll}\mathrm{X} & \mathrm{Y}\end{array}\right] \mathrm{Z}$

,Herr, der macht, dass der Himmel mit Regen (versehen) ist ${ }^{\text {"11 }}$

In Bsp. (5) ist in der externen Relation Z (páti-) der Partizipant, der veranlasst, dass Y (-dyu-) X (mit Regen) ist oder wird. ${ }^{12} \mathrm{Y}$ entspricht in der inneren Relation dem Subjekt einer Kopulaprädikation und

9 5.8.6cd: urujráyasaṃ ghrtáyonim áhutam tveșám cákșur dadhire codayánmati ,Zu dem, der weiten Raum schafft, dessen Stätte (reich an) Schmelzbutter ist, der begossen wird, zu ihrem funkelnden Auge, das das Denken anregt, haben sie (die Götter dich, Agni,) gemacht'; ghrtáyoni- findet sich noch in 3.4.2 (Tanūnapāt), 5.68.2 (Mitra und Varuṇa).

$10 \mathrm{~S}=$ alleiniges Argument eines intransitives Verbs; $\mathrm{A}=$ agenshaftes Argument eines transitiven Verbs; $\mathrm{P}=$ weniger agenshaftes Argument eines transitiven Verbs.

11 5.68.5: vrștídyāvā rītyà peșás pátī dánumatyāh | brhántam gártam āśāte ,Sie beide (Mitra und Varuṇa), die machen, dass der Himmel Regen hat, dass die Wasser fliessen, die zwei Herren des tropfenreichen Lebenssafts haben den hohen Sitz erlangt.' Vgl. noch 9.106.9 da, auch neben rītyà̀ - -, von den Somatropfen. Die beiden Bildungen hat Schindler in den $80 e r n$ in Vorträgen und im Unterricht in die Diskussion eingebracht, vgl. Schindler (1997, 539); Janda (1999, 202f.); Ramharter (2012, 274).

12 Dem für Kasus unspezifizierten Vorderglied vrșțí- des Kompositums entspricht funktional und formal in einem freien Syntagma ein Instrumental bzw. die Ableitung auf -mat- in vrșțimát- (Schindler 1986, vgl.). Eine verbale Interpretation 
$\mathrm{X}$ dem Prädikat(snomen). Eine kausative Interpretation impliziert immer eine prädikative interne Relation (S. 8ff.).

Die kausative Relation ist von der Relation des ersten Typs (Possessive Modifikation, S. 4f.) formal nicht unterschieden, so dass die zutreffende Lesart sich ausschliesslich aus der Semantik der beteiligten Begriffe $\mathrm{X}, \mathrm{Y}$ und $\mathrm{Z}$ in einem konkreten Kontext ergibt, und oftmals ist mehr als eine Lesart möglich bzw. keine a priori ausgeschlossen. Eine kausative Lesart ist deshalb für das Vedische nur selten mit Sicherheit feststellbar, obwohl sie an vielen Stellen möglich und sinnvoll ist. Allerdings sind solche kausative Relationen aus kontextuellen und semantischen Gründen in vergleichbaren avestischen (s. Bsp. 6) und griechischen (Bsp. 7) Komposita sicher anzunehmen. Dieser Umstand legt nahe, dass diese kausative Relation eine ererbte Eigenschaft des Kompositionstyps ist und auch im Vedischen zur Verfügung steht, wenn auch möglicherweise in geringerem Umfang genutzt wird.

(6) pantąm [pasnuu- ̊̊nhom ][hikuu- ånham ] Pfad.AKK.sG [Staub Mund.AKK.sG ] [trocken Mund.AKK.sG ] ,Pfad, der macht, dass der Mund mit Staub versehen ist, dass der Mund trocken ist ${ }^{13}$

Da ein Pfad keinen Mund hat, ist die kausative Lesart der externen Relation in (6) die eindeutig beste Interpretation.

Im Griechischen werden die kausative Lesart und die Lesart des ersten Typs auch ganz gezielt zur Erzielung stilistischer Effekte eingesetzt.

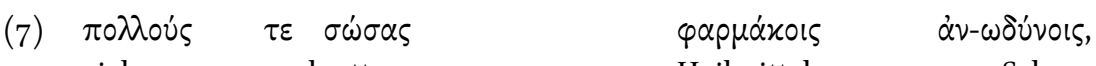
viel.AKK.PL.M und retten.AOR.PART.NOM.SG.M Heilmittel.DAT.PL.N NEG-Schmerz.DAT.PL.N

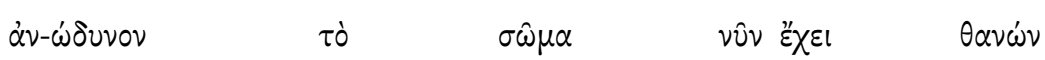

NEG-Schmerz.AKK.SG.N ART.AKK.SG.N Körper.AKK.SG.N nun haben.PS.3SG sterben.AOR.PART.NOM.SG.M

,Und nachdem er vielen mit schmerzlindernen Mitteln geholfen hat,

hat er im Tod nun einen Leib ohne Schmerzen. ～～～(Epitaph eines Arztes, IG14.1879)

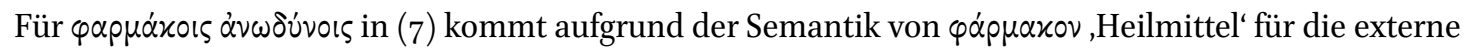
Relation nur die kausative Lesart ,mit Heilmitteln, die machen, dass die Schmerzen nicht (mehr) existieren' (,allaying pain' LSJ ${ }^{9}$, s. v.) in Frage.

,den Himmel regnen lassend' (Lühr 2004, 68), ,bringing the heavens to rain' (Jamison \& Brereton 2014, 3, 1353) ist nicht auzuschliessen, doch hat vrșțí- synchron die konkrete Bedeutung ,Regen. Vgl. aber immerhin in derselben an Mitra und Varuṇa gerichteten Liedergruppe die Stellen 5.63.3d: dyám varșayathas und 5.63.6d: dyám varșayatham ,ihr lasst/lasst den Himmel regnen' und desweiteren die Ausführungen bei Wackernagel $(1905,320)$.

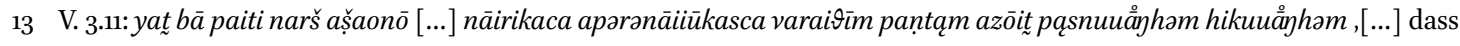
von einem rechtschaffenen Mann weg [...] (seine) Frau und auch (sein) noch nicht volljähriges Kind auf dem Pfad in die Gefangenschaft zieh(en, auf dem Pfad, der) den Mund mit Staub füllt, den Mund austrocknet.' Zur Interpretation von pasnuu-ăgh 


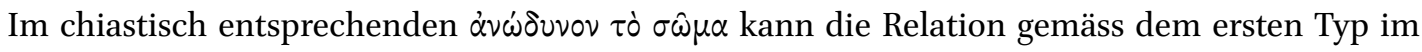
Sinne von ,schmerzlos' interpretiert werden, d. h. die externe Relation kann mit ,Schmerz des Körpers paraphrasiert werden und interne Relation mit ,der Schmerz ist/existiert nicht', (,free from pain' LSJ ${ }^{9}$, s. v.). Interessanterweise existiert hier auch die Lesart, die die kausative externe Relation negiert: der Körper macht nicht (mehr), dass Schmerz existiert' (,causing no pain` LSJ ${ }^{9}$, s. v.). Bemerkenswert ist in diesem Fall, dass die morphologisch im Vorderglied des Kompositums vorhandene nominale Negation $(\dot{\alpha} \nu$-) Skopus über die externe kausative Relation hat. Im Verhältnis zur Lesart ,schmerzlos' kann man von einem Transfer der Negation sprechen.

Im Vedischen entspricht mit vergleichbaren semantischen Bestandteilen das Kompositum anamīvá-, s. Bsp. (8).

(8) [an- amìvás ] ] ș̣as

[NEG- Krankheit.NOM.PL.F ] Labungen.NOM.PL.F

,die Labungstränke, die verursachen, dass die Krankheiten nicht (mehr) existieren ${ }^{14}$

\section{S/A-Verb-Relation}

Im dritten Typ der externen Relation entspricht das Verhältnis zwischen Z und Y dem Verhältnis zwischen einem S/A-Argument (Z) und einem Sachverhalt, der in einem der beiden Kompositionsglieder (meistens Y) als verbales Konzept ausgedrückt wird. ${ }^{15}$ Die S/A-Funktion der Handlung wird dabei systematisch von $\mathrm{Z}$ wahrgenommen, vgl. (9)-(10).

(9) [ánna-kāma- ] grhú-

[ Speise VERLANGEN ] Bettler

$\left[\begin{array}{lll}\mathrm{X} & \mathrm{Y}\end{array}\right] \mathrm{Z}$

,der Bettler verlangt' $\sim$,das Verlangen des Bettlers ${ }^{\mathrm{a} 6}$

14 3.62.14: sómo asmábhyaṃ dvipáde cátușpade ca paśáve | anamivaá ișas karat ,Soma soll uns zuliebe für Gesinde und Vieh (wörtlich: ,für das zwei- und vierbeinige Vieh') Stärkungen, die Krankheiten fernhalten, beschaffen` bzw. ,soll machen (karat), dass die Nahrung keine Krankheiten verursacht'. Vgl. noch mit kausativer Lesart 3.22.4 (ișas), 10.17.8 (ișas), 10.98.3 (vác-), dann 7.46.2d: anamivó rudra jásu no bhava ,Werde, Rudra, vor unseren Sprösslingen einer, der Krankheiten fernhält!', ähnlich 7.54.1 von vástoṣ pati- (etwa ,Lar') und 10.35.6 von den uṣásas. Für nicht kausative Belege vgl. 3.16.3 (suvíryaoder doch rayí-?), 3.59.3 (vayám), 10.18.7 (jánayas), 10.37.7 (,wir') und, substantiviert, 10.14.11. Im Falle von Rudra, der als Verbreiter von Seuchen und Krankheiten gilt, diese jedoch auch entziehen und somit heilen kann, ist der oben im Text angesprochene Transfer der Negation greifbar: ,ür uns wird/sei einer, der (entgegen deinem Naturell) nicht macht, dass Krankheiten existieren' (Verbreiter von Seuchen) vs. , für uns wird/sei einer, der macht, dass Krankheiten nicht (mehr) existieren' (Heiler und Prophylaktiker).

15 Ein verbales Konzept unterscheidet sich hier von einem nicht-verbalen Konzept insofern, als es innerhalb des Vedischen mit einer verbal ausgedrückten Handlung assoziiert werden kann (z. B. -kāma- mit $k \bar{a}-$, verlangen‘ [Perf. cake] vs. nichtverbal śátru- ,Feind'). In vielen Fällen ist eine Unterscheidung zwischen verbal und nicht-verbal nicht notwendig, da dies die Semantik des Ausdrucks nicht tangiert.

16 10.117.3ab: sá íd bhojó yó grháve dádāty ánnakāmāya cárate krśááya ,Der wahrlich ist freigebig, der einem Bettler gibt, (einem Bettler,) der begierig nach Speise (und) abgemagert einherwandelt.' 
(10) [idhmá- bhrti- ] dabhíti-

[ Feuerholz BESORGEN ]

$\left[\begin{array}{lll}\mathrm{X} & \mathrm{Y}\end{array}\right] \mathrm{Z}$

,Dabhīti (EN) besorgt' $\sim$,das Besorgen des Dabhīti $(\mathrm{EN})^{{ }^{a 7}}$

In (9) ist das Kompositionshinterglied Y eine nominale Verbform der Bedeutung, Verlangen', Z hat die A-Funktion und X übernimmt die P-Funktion in der nominal ausgedrückten Verbalhandlung.

Die Übergänge zwischen dem ersten Typ (S. 4f.) und dem hier besprochenen dritten Typ sind insofern nicht scharf, als die externe Relation des dritten Typs hier wie die Relation des ersten Typs als Modifikation interpretiert werden kann, so wie das in den Beispielen (,das Verlangen von Z' in Bsp. (9) oder ,das Bereitstellen des Z' in (10)) angedeutet ist. Die Verfügbarkeit beider Interpretationsarten der externen Relation ist aber nur für externe Relationen des dritten Typs gegeben. Für den ersten Typ (vgl. S. 4 mit Bsp. (3) tigmá́yudha-) ist die Interpretation nach dem dritten Typ nicht ohne weiteres verfügbar, da im Kompositum kein verbales Konzept explizit vorhanden ist. Eine besondere Affinität zum dritten Typus haben im übrigen die synthetischen Komposita. ${ }^{18}$

\section{Tabellarische Übersicht}

Die wesentlichen Eigenschaften der drei Typen externer Relationen sind in der Tab. 1 zusammengefasst.

\begin{tabular}{llll}
\hline & \multicolumn{1}{c}{ Typ 1 } & \multicolumn{1}{c}{ Typ 2 } & \multicolumn{1}{c}{ Typ 3 } \\
\cline { 2 - 4 } Art der Relation & Possession & Kausativ & S/A-Verb \\
Ausdruck der Relation & implizit & implizit & lexikalisch in Y \\
Funktion von Z & Possessor & causer & S/A \\
Funktion von $\mathrm{Y}(/ \mathrm{X})$ & Possessum & causee & verbales Konzept \\
\hline
\end{tabular}

Tabelle 1: Wesentliche Eigenschaften der externen Relation $(\mathrm{Z} \leftrightarrow \mathrm{Y}(/ \mathrm{X}))$ nach Typen

\section{Interne Relation}

\section{Kopulaprädikation}

In einem ersten Typ ist die Relation zwischen X und Y so geartet, dass das Kompositionsglied, das nicht an der externen Relation Teil hat (meist X), als Prädikat von Y in einer Kopulaprädikation fungiert

17 6.20.13cd: dīdáyad ít túbhyạ̣ sómebhih sunván dabhítir idhmábhrtiḥ pakthy àrkáih ,Erstrahlt sein wird er durch die dir (zugedachten) Somasäfte, schon während er (sie) presst, der Dabhīti, (und wenn) er unter Preisgesängen das Brennholz besorgt und Speisen gekocht hat.' Vgl. für idhmá- + bhr- etwa in 1.94.4a: bhárāmedhmám ... te ..., wir wollen dir Brennholz bringen:

18 Vgl. zum Begriff Schroeder (1874, 206ff., 287ff.), Wackernagel (1905, 174 mit Lit.) und nun auch Nielsen Whitehead (2012, 9, 27-39). Für diese Komposita ist die Interpretation der externen Relation als Modifikation sehr unwahrscheinlich, wenn nicht gar ganz auszuschliessen. 
(s. Bsp. 11). Diese Kopulaprädikation wird in der internen Relation morphosyntaktisch nicht explizit ausgedrückt.

Von der Frequenz her sind die Lesarten, die als Prädikation mit der Kopula AS ,sein' interpretierbar sind, am geläufigsten. Die konkret möglichen Lesarten ergeben sich aber auch hier aus dem Kontext, aus der Semantik und aus den Eigenschaften der beteiligten Partizipanten und Sachverhalte.

In (11) wird so der Feind (-śatru-, Y) von Vrrtra als Indra (X) identifiziert (,der Feind von Vrtra ist Indra $)^{\prime}$.

(11) [índra-śatru- ] vortrá-

[ Indra Feind ] Vrrtra

$\left[\begin{array}{lll}\mathrm{X} & \mathrm{Y}\end{array}\right] \mathrm{Z}$

,Vrtra, dessen Feind Indra ist ${ }^{19}$

Es kommen auch andere Arten von Prädikaten vor, wie die folgenden Beispiele zeigen. Bei lokativischen Prädikaten kann dabei ausnahmsweise die lokativische Semantik durch eine Kasusendung am Kompositionsvorderglied morphosyntaktisch ausgezeichnet werden, vgl. (12a) mit dem Vorderglied āsán ,im Mund ${ }^{20}$ Ein mögliches Übersetzungsäquivalent der internen Relation in (12a) ist, der Pfeil/die Pfeile ist/sind im Mund'.

(12) a. [äsánn- ișu- ] gav-

[ Mund.Lok- Pfeil ] Rind

$\left[\begin{array}{lll}X & Y\end{array}\right] \mathrm{Z}$

,Rinder (des Rata), deren Pfeile in (ihrem) Munde sind ${ }^{21}$

b. [issu- hasta- ] índra-

[ Pfeil- Hand ] Indra

$\left[\begin{array}{lll}\mathrm{X} & \mathrm{Y}\end{array}\right] \mathrm{Z}$

,Indra, dessen Hand voller Pfeile ist ${ }^{22}$

In (12b) kann die interne Relation zwischen der Hand und den Pfeilen mit, die Hand ist mit Pfeilen' paraphrasiert werden, in dem das Kompositionsvorderglied die Funktion eines prädikativen Instrumentals übernimmt. Schindler $(1986,395)$ bezeichnet diesen Bildungen als Doppelpossessiva. Gleichzeitig

19 1.32.6: nátāiēd asya sámritim vadhánām sám rujánāḥ pipișa índraśatruḥ ,Nicht hielt er (Vṛtra) dem Ansturm seiner (Indras) Waffen stand; mit zerbrochener Nase (?), zermalmt (lag) er da, er, dessen Feind Indra war/der sich Indra zum Feind gemacht hatte' $S$. auch im selben Hymnus den zweiten Beleg in 1.32.10.

$20 \mathrm{Zu}$ den belegten Kasus in Vordergliedern von Komposita Wackernagel (vgl. 1905, 201ff.; 277ff.), speziell zu denen von Wurzelkomposita Scarlata (1999, 743ff.)

21 1.84.16: kó adyá yunkkte dhurí gár rtásya ... āsánnișūn hrtsváso mayobhún ... ,Wer schirrt heut die Rinder des Rota ans Joch ..., die ihre Pfeile im Munde führen, die (damit) in die Herzen treffen (und doch) zur Freude gereichen ...' (die Rede ist von den Priestern, die Pfeile sind ihre Worte).

22 10.103.2cd: tád índrena jayata tát sahadhvam yúdho nara ișuhastena vŕ̛ș̣nā, Nun seid gemeinsam mit Indra siegreich, nun gewinnet die Kämpfe, Männer, gemeinsam mit dem Bullen, dessen Hand (schon) voller Pfeile ist'. In der gleich folgenden Roc findet sich der zweite Beleg (ișuhastais neben nișañgíbhis etwa ,Köcherträger'), auf Indras Mannen (den Maruts) bezogen; vgl. inhaltlich entsprechend auch von den Maruts 1.64.10c: ástāra ișuṃ dadhire gábhastyor ,die Schützen halten (je) einen Pfeil in ihren Händen'. 
weist er darauf hin, dass in solchen Fällen auch lokativische Syntagmen als finite Entsprechnungen zur Verfügung stehen (vgl. rukmá-vakșas- ,Schmuck auf der Brust habend' : vákșassu rukmáh ,auf der Brust ist Schmuck' RV 1.166.10), wobei das lokativische Prädikat als Y auftritt und X die S/A-Funktion bekleidet. Komposita wie in (12b) verfügen in diesem Sinn systematisch über eine lokativische und eine instrumentale Lesart. ${ }^{23}$

Weiter kommen auch Lokaladverbien als Prädikate vor.

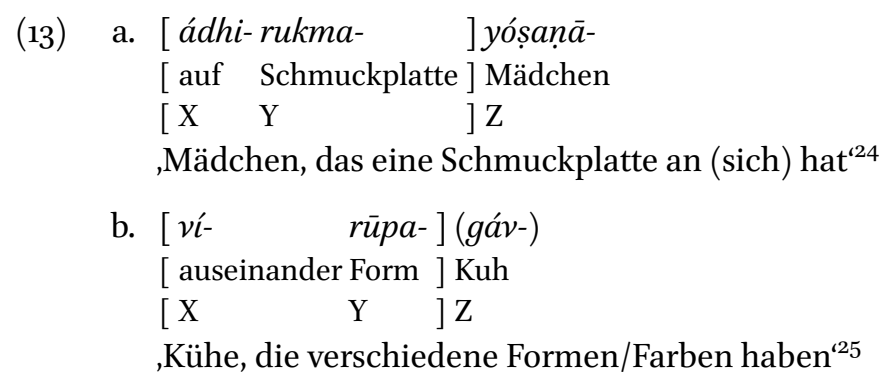

In (13a) bildet ein Lokaladverb das Prädikat und die interne Relation kann paraphrasiert werden mit ,die Schmuckplatte ist darauf (sc. auf dem Mädchen)`. Das Lokaladverb ádhi lokalisiert somit rukmá„Schmuckplatte‘ (Y) auf yóṣaṇā- (Z) , wodurch zusätzlich ein Rückbezug auf yóṣ̣aṇā- (Z) hergestellt wird, das ausserhalb der internen Relation steht.

Bsp. (13b) unterscheidet sich insofern von (13a), als das Adverb vi-im Kompositionsvorderglied in keinem Bezug steht zu einem Partizipanten ausserhalb der internen Relation. Die interne Relation kann in diesem Beispiel mit ,die Formen/die Form sind/ist auseinander (i. e. verschieden) ‘ paraphrasiert werden. ${ }^{26}$ In einem Untertyp kann die interne Prädikation vielleicht auch als kausativ mit $Y$ als causer

23 Die lokativische Lesart ist hierbei vielleicht sekundär, aber durch die finiten Entsprechungen synchron klar gestützt. Im Vergleich mit dem Beispiel in (12a) kann der Eindruck entstehen, die Reihenfolge der Kompositionsglieder sei frei, sind doch beide Komposita prima facie interpretierbar als ,mit Pfeilen in X'. Der Unterschied besteht jedoch lediglich darin, dass im einen Fall über die Hand eine Aussage gemacht wird und im anderen Fall über die Pfeile. Das Phänomen ist noch nicht abschliessend beschrieben worden und ist im Übrigen nicht direkt mit den in Wackernagel (1905, 302ff.) beschriebenen „Abnormitäten“ vergleichbar.

24 8.46.33: ádha syáyóṣaṇa mahí pratīcíváśam aśryám |ádhirukmā ví nāyate \|, Und dann noch diese junge Frau, die grosse, die gegenüber von Vaśa Aśvya, mit einer Schmuckplatte an (sich) wird sie von dannen geführt.' Für ein dem Kompositum entsprechendes Syntagma vgl. 1.64.4b, 8.20.11b, dann als weiteres Beispiel in einer vergleichbaren Begriffssphäre ádhi-vastra- ,mit einem (Fest-)Kleid an' in 8.26.13 von einer Braut bzw. jungen Frau (vadhú-).

25 10.169.2: yấh sárūpā vírūpā ékarūpa yásām agnír iștyā nấmāni véda | yáa ángirasas tápasehá cakrús tábhyah parjanya máhi śárma yacha \| ,Die gleichfarbig, verschiedenfarbig (und doch) von einer Gestalt sind, deren Namen Agni durch die Opfertätigkeit kennt, die die Angirasen mit Eifer hierher geschafft haben, für die, Parjanya, spanne deinen grossen Schirm auf.' vírūpa- $(17 \times)$, oft von Nacht und Morgen, wo auch eine kausative Lesart nicht unwahrscheinlich ist. S. auch Sadovski (2000, 465f. bzw. 457).

26 Von den Komposita in (13a) und (13b) zu unterscheiden sind solche, bei denen X als Präposition oder präpositionelles Adverb Y regiert und weitere Relationen zwischen Z und X/Y von den o. (S. 4f.) behandelten verschieden sind, vgl. z. B. adhoakșá- von den Flüssen in 3.33.9cd: bhávatā ... adhoakșăh sindhava h srotyábhih ,bleibet, ihr Flüsse, unter der Achse (unserer Wagen) mit euren Strömen'. Dass es sich hierbei um die Achsen von Fahrzeugen handelt, und nicht etwa um die Achseln der Flussdurchquerer, erhellt aus der vorausgehenden Verszeile 3.33.9b: yayáu vo dūrád ánasā ráthena ,er 
und $\mathrm{Z}$ als causee interpretiert werden, ${ }^{27}$ doch sind diese Fälle auch anders deutbar.

Als Kompositionsvorderglied kann auch die nominale Negation $a$-verwendet werden. In diesen Fällen wird in der Regel wie in (14) die Existenz von Y negiert.

(14) [a- pád- $]$ Vrtra-

[ NEG- Fuss ] Vrtra-

$\left[\begin{array}{lll}\mathrm{X} & \mathrm{Y}\end{array}\right] \mathrm{Z}$

,der Fuss/die Füsse des Vrrtra existiert/en nicht ${ }^{28}$

\section{Modifikation}

In einem zweiten Typ besteht zwischen den beiden Gliedern der internen Relation ein Modifikationsverhältnis.

(15) [ váta- raminhas- ] rátha-

[ Wind Geschwindigkeit ] Wagen

$\left[\begin{array}{lll}\mathrm{X} & \mathrm{Y}\end{array}\right] \mathrm{Z}$

,windschneller Wagen ${ }^{29}$

(16) [áyah-sthūnāa- ] gárta-

[ Erz Säule ] Hochsitz

$\left[\begin{array}{lll}X & \mathrm{Y}\end{array}\right] \mathrm{Z}$

,Säule aus Erz habender Hochsitz ${ }^{30}$

ist aus der Ferne zu euch mit Last- und Streitwagen gefahren gekommen. Für weitere Beispiele vgl. Wackernagel (1905, 308ff.) und in extenso Sadovski (2000).

27 So vielleicht in mitájinu- (3.59.3, 6.32.3, 7.82.4, 7.95.4), falls das Kompositionsvorderglied ,aufgerichtet' bedeutet (und nicht einfach ,fest, fix', wie ein Gutachter moniert). An allen vier Stellen wird mit dem Kompositum jedenfalls die aufrechte Körperhaltung als Achtungsstellung bei der Ehrerbietung angesprochen. Dem entspricht auch ürdhvá-jñu- in MS I 10.9 (4); vgl. Amano (2009, 369f., Fn. 1332) sowie Sadovski (2000, 466) mit Lit. In ūrdhvá-jñu-ist die Bedeutung des Kompositionsvorderglieds wohl weniger umstritten. Da die Vorstellung von ,aufrechten, aufgerichteten, in die Höhe ragenden Knien' allenfalls beim Sitzen in der Hocke Sinn ergibt oder beim Liegen auf dem Rücken mit angewinkelten Beinen, kaum aber beim Stehen, ist die Interpretation zumindest von ūrdhvá-jñu- ,mit Knien, die machen, dass Z aufrecht ist/steht' semantisch in Betracht zu ziehen. Vgl. als weiteres mögliches Beispiel citrá-śociș- vom Nachthimmel in 5.17.2: ,mit glänzenden Lichtern` oder mit einer kausativen Interpretation ,mit Lichtern, die ihn (den Nachthimmel) glänzend machen'? Dann etwa pūtádakșa- z. B. von den Maruts in 5.29.1, von reiner Gesinnung' oder kausativ ,deren (ehrenvolles) Tun darin besteht, rein zu machen'? S. noch das aussergewöhnliche, dreigliedrige raghupátmajaṃhas- in 6.3.5 von Agni im Vergleich mit einem Vogel ,dessen Schwung/Flug durch (sein) Gefieder schnell wird ' bzw. ,dessen Gefieder macht, dass dessen Flug schnell ist/wird".

28 1.32.7ab: apád ahastó aprtanyad índram ásya vájram ádhi sánau jaghāna, Obwohl er (Vrrtra) keine Füsse hatte, obwohl er keine Hände hatte, kämpfte er gegen Indra an: der (Indra) hat auf dessen Rücken den Vajra (darauf) gehauen' Weiter wird apád-auch in 3.30.8 von Vrrtra gesagt, von einem weiteren dämonischen Wesen (Atra) in 5.32.8 und in weiteren sieben Belegen von Ușas, Agni, Himmel und Erde etc.

29 5.77.3: híranyatvañ mádhuvarṇo ghrósásuh próksọo váhann á rátho vartate vām | mánojavā aśvinā vấtaraṃhā yénātiyāthó duritấni viśva a ||,Mit goldener Plane, honigfarben, mit Schmalz bedeckt, rollt, indem er Stärkungen führt, euer Wagen heran; gedankenschnell, Aśvins, windeseilig (ist er), mit dem ihr über alle Unwegsamkeiten hinwegfahrt.; vom Wagen der Aśvins auch in 1.118.1, dann von den Rossen der Aśvins in 1.181.2 und überhaupt von Rossen in 8.34.17.

30 5.62.8: híranyarūpam uṣáso vyùșțāv áyahsthūnam úditā súryasya |á rohatho varuṇa mitra gártam átaś cakșāthe áditim dítị ca $\mid \|$,Den goldfarbigen beim Aufleuchten der Ușas, den mit der Erzsäule beim Abgang der Sonne besteigt ihr, Varuṇa, 
In (15) modifiziert vâta- (X) das Hinterglied -ramihas- (Y) und kann mit ,Geschwindigkeit des Windes paraphrasiert werden. Wenn es sich wie in diesem Beispiel (15) bei Y um ein Adjektivabstraktum handelt (hier rámhas), ergibt sich als Gesamtbedeutung des Kompositums diejenige eines vergleichenden adjektivischen Determinativkompositums (hier ,schnell wie der Wind').

In Beispiel (16) entspricht die Modifikation des Y (sthúnāā) durch X (áyas-) funktional einem Genitivus materiae. $3^{1}$

Wenn das Kompositionshinterglied ein verbales Konzept ausdrückt und das Vorderglied nichtsubstantivisch ist, ergibt sich bei intransitiven Sachverhalten zwangslos eine adverbial modifizierende Interpretation des Kompositionsvorderglieds wie in (17). Die Funktion entspricht der eines partizipialen Determinativkompositums.

(17) [ raghu-yáman- ] sóma-

[ schnell EILEN ] Soma

$\left[\begin{array}{lll}\mathrm{X} & \mathrm{Y}\end{array}\right] \mathrm{Z}$

,schnell eilender Soma ${ }^{32}$

Für substantivische Kompositionsvorderglieder, die als Adjunkte fungieren, vgl. mit expliziter Kasusmarkierung etwa mit dem Lokativ apsu-já- und divi-já-, in den Wassern` bzw. ,im Himmel geboren (8.43.28), mit dem Instrumental hräduni-vŕt-, mit Hagel (heran)rollend' (5.54.3) und mit dem Ablativ divo-rúc-, vom Himmel her leuchtend' (wenn nicht,Himmelsleuchte' 3.7.5). ${ }^{33}$ Mit apsu-já́- vergleicht sich ohne Kasusmorphologie $a b-j a ́$ - (4.40.5, 7.34.16); derartige Doubletten sind wiederkehrend, so z. B. auch rote-já́- und $r t a-j a ́$ -

In vielen Fällen ist dieser zweite modifikative Typ vom ersten prädikativen Typ (S. 8ff.) nicht unterscheidbar. Beide Typen stellen lediglich mögliche Interpretationsmuster zur Verfügung. In der Regel sind beide Muster verfügbar, wenn im Kompositionsvorderglied ein Lexem steht, das wie tigm(á)-in (18) als Adjektiv aufgefasst werden kann.

(18) [ tigm(á)-āyudha- ] índra-

[ scharf Waffe ] Indra

$\left[\begin{array}{lll}\mathrm{X} & \mathrm{Y}\end{array}\right] \mathrm{Z}$

,Indras [Waffe ist/wird scharf]

$\sim$,Indras [scharfe Waffe]

Mitra: den hohen Sitz. Von da aus haltet ihr Ausschau nach Aditi und Diti.; damit vergleicht sich in der vorhergehenden Roc 5.62.7a: híranyanirnig áyo asya sthứna ā ,mit Gold ummantelt, (aus) Erz (ist) dessen Säule mit der Form áyas, die für den haplologisch verkürzten Genitiv áyasas (wozu gleich in der nächsten Fn.) vor asya stehen könnte: genauso auch in 1.163.9a vom idealisierten Pferd: ... áyo asya pádā, (aus) Erz sind dessen Hufe‘. Angesichts von yásyā áyo múkham in 6.75.15b ,(Pfeil), dessen „Mund“ Erz ist', woran sich áyahśírșan- in 8.101.3 (vom Pfeil als Boten von Varuṇa und Mitra) anschliessen lässt, ist die Annahme einer Haplologie (áyas für áyasas) entbehrlich.

31 S. áyaso ná dhấrām ,wie eine Klinge aus Erz' in 6.3.5 und 6.47.10.

32 9.39.4: ayám sá yó divás pári raghuyámā pavítra á | síndhor ūrmá vy ákșarat \| ,Der hier ist es, der vom Himmel her (kommend) in rascher Fahrt auf der Seihe, in einer Woge (wie die) eines Stromes fliessend sich aus(breitete).

33 Für weitere Komposita mit kasuellem Kompositionsvorderglied s. Scarlata (1999, 743ff.) und jeweils die Diskussionen s. vv. 
Bsp. (18) kann so interpretiert werden, dass tigmá- (X) funktional dem Prädikat und áyudha- (Y) dem S-Argument einer Kopulaprädikation mit AS (,die Waffe ist scharf') oder BHŪ (,die Waffe wird scharf') entspricht. Das Verhältnis zwischen den beiden Kompositionsgliedern kann jedoch auch als Modifikation angesehen werden (,scharfe Waffe').

Welche Interpretation allgemein die passende ist, ergibt sich aus dem Zusammenspiel zwischen den Kompositionsgliedern und dem modifizierten Begriff in einem spezifischen Kontext. Bei Komposita mit Lokaladverbien im Vorderglied z. B. ist die prädikative Interpretation der internen Relation (ádhi-rukma,Schmuck ist darauf [sc. auf/an Z]') jeweils passender.

Die Interpretation der internen Relation wird auch von der externen Relation gesteuert: Wenn die externe Relation kausativ interpretiert wird, ist die interne Relation prädikativ zu verstehen (,Z macht, dass $\mathrm{YX}$ ist/wird').

\section{Verb-Argument}

Im dritten Typ besteht die Relation zwischen X und Y in einem Rektionsverhältnis, in dem Y ein verbales Konzept ausdrückt und $\mathrm{X}$ ein Argument davon ist (seltener vice vers ${ }^{34}$ ). Am durchsichtigsten und häufigsten sind P-Argumente im Vorderglied, vgl. (19).

(19) [ánna-kāma- ] ] grhú-

[ Speise VERLANGEN ] Bettler

$\left[\begin{array}{lll}\mathrm{X} & \mathrm{Y}\end{array}\right] \mathrm{Z}$

,Bettler, der Speise verlangt‘

Das Kompositionsvorderglied entspricht in einem finiten Ausdruck dem direkten Objekt des transitiven Sachverhalts. Geeignete Übersetzungsäquivalente sind oft Partizipialkonstruktionen wie ,Speise verlangend'. Dass die Vorderglieder in diesen Fällen wie direkte Objekte finiter Konstruktionen verstanden wurden und diese Komposita als infinite Satzentsprechungen verwendet werden konnten, suggeriert Bsp. (20). In dieser Korrelativkonstruktion steht im Relativsatzsegment das Relativpronomen als Vorderglied in einem Kompositum.

(20) yát-kāmās te juhumás tán no astu

REL.AKK.SG.N-erheischen.NOM.PL dir opfern.IPL.PS dies.AKK.SG.N uns sein.IMP.3SG

,Was wir mit unserem Opfer für dich zu erlangen suchen, das soll unser sein!‘

(Wörtlich: ,[Was erheischend] wir dir opfern, das soll unser sein!') 10.121.10c

Als Argument des Kompositionshinterglieds -kāma- leistet yát- hier zusätzlich die subordinierende Funktion für das finite Verb, was eine bemerkenswerte Konstellation darstellt. ${ }^{35}$

34 Z. B. sanád-vāja- Siegpreise (-vāja-) erringend' und Ähnliches, vgl. Dunkel (1992, 197-209), Schindler (1997, 539) und nun in extenso Nielsen Whitehead (2012). Die Reihenfolge ist hier nicht weiter von Belang.

35 S. auch Schindler $(1997,538)$; vgl. weiter ŚB 1.6.2.12.7 mit einer analogen Konstruktion, die auf dieser Stelle zu basieren scheint. 
Während yát- in yát-kāma- als kasusneutrale Kompositionsform interpretierbar ist und nicht unbedingt als Kasusform angesehen werden muss, ${ }^{36}$ wird in anderen Bildungen das verbale Rektionsverhältnis teilweise explizit gemacht, indem X als direktes Objekt in den Objektkasus gesetzt wird, vgl. mit dem Hinterglied -kāma- aus Bsp. (20) noch tvấn-kāma- (8.11.7c ágne tvấmkāmayā giráa ,... mit einem Lied, Agni, das nach dir verlangt'), ferner mit ambigen Vordergliedern śrávaskāma- (Stamm oder Akk. Sg.) in 8.2.38 und rāyáskāma- (Gen. Sg. oder Akk Pl.) in 1.78.2, 7.32.3, 7.42.6. Die Verwendung einer overten Kasusform für direkte Objekte in diesen Kompositionstypen wird diachron im Vedischen nach und nach beschränkt auf Komposita mit Hintergliedern der Form R(a)- $a$ - wie in Bsp. (21). Diese explizite verbale Rektion korreliert mit entsprechenden verbalen Eigenschaften dieser Formen als Simplizia. Zudem ist bei Hintergliedern, die eindeutig auf einen Verbalstamm zurückgehen, die Kasusmarkierung am direkten Objekt nach Schindler $(1997,537$ f.) obligatorisch.

(21) [dhanaṃ- jayá- ] agní-

[ Schatz.AKK.SG ERRINGEN ] Agni

$\left[\begin{array}{lll}\mathrm{X} & \mathrm{Y}\end{array} \mathrm{Z}\right.$

,Agni, der Schätze erringt ${ }^{‘ 37}$

Im Kompositionsvorderglied erscheinen nebst direkten Objekten auch andere Argumente, z. T. mit overter Kasusform. Dazu gehören Lokative wie in apsu-kṣít- ,in den Wassern weilend' (1.139.11) oder divi-kșít- ,im Himmel weilend' (10.92.12), woran sich das gleichbedeutende divi-kșayá- (5.46.5) anschliesst. $^{38}$

\section{Tabellarische Übersicht}

Die wesentlichen Eigenschaften der drei Typen interner Relationen sind in der Tab. 2 zusammengefasst.

\begin{tabular}{llll}
\hline & \multicolumn{1}{c}{ Typ 1 } & \multicolumn{1}{c}{ Typ 2 } & \multicolumn{1}{c}{ Typ 3 } \\
\cline { 2 - 4 } Art der Relation & Kopulaprädikation & Modifikation & Verb-Argument \\
Ausdruck der Relation & implizit & implizit & \pm explizit \\
Funktion von Y & Subjekt der Präd. & Modifikatum & Verbales Konzept \\
Funktion von X & Prädikat & Modifikator & Argument \\
\hline
\end{tabular}

Tabelle 2: Wesentliche Eigenschaften der internen Relation $(\mathrm{X} \leftrightarrow \mathrm{Y})$ nach Typen

36 S. Sadovski (2002, 372-380, insbes. 375-379, mit Lit.).

37 1.74.3: utá bruvantu jantáva úd agnír vrtrahájani | dhanaṃjayó ráne-raṇe $\|$,Und Leute und Stämme (allüberall) sollen sagen: Agni, der Zerschlager von Schranken, ist auferstanden, (er), der Schätze in auch jedem Wettkampf erringt.' Von Agni auch in 6.16.15, dann von Indra (3.42.6, 8.45.13) und Soma (9.46.5, 9.84.5). Vgl. auch mit gleicher Bedeutung dhanajit(s. Scarlata 1999, 156)

38 Mit thematischem Hinterglied finden sich noch in 7.55.7 proșthe-śayá- und vahye-śayá- (von Frauen:) ,auf einem Feldbett bzw. ,auf einer Sänfte liegend' neben talpa-ść-varì- (f.) , auf einem Diwan liegend', das seinerseits mit talpe-śayá-in AV 4.5.3 korrespondiert. 


\section{Diskussion und Zusammenfassung}

Von den drei Relationen zwischen den Elementen eines Syntagmas bestehend aus einem Bezugsnomen und einem attributiven exozentrischen Kompositum wurden hier die semantisch-syntaktischen Eigenschaften der externen Relation (zwischen einem einzelnen Kompositionsglied und dem Bezugsnomen) und der internen Relation (zwischen den beiden Kompositionsglieder) untersucht und für beide Relationen sind drei unterschiedliche Ausprägungen ausgemacht worden.

Rechnerisch existieren neun Möglichkeiten, wie diese Relationen kombiniert werden können, doch sind nicht alle möglich bzw. es werden nicht alle Möglichkeiten ausgeschöpft, s. gleich Tab. 3:

\begin{tabular}{l|ccc}
\hline \multicolumn{1}{c|}{ extern } & Possession & Kausativ & S/A-Verb \\
intern & + & + & - \\
\hline Kopulaprädikation & + & - & + \\
Modifikation & - & - & + \\
Verb-Argument & - & - & + \\
\hline
\end{tabular}

Tabelle 3: Kombinationsmatrix externer und interner Relationen (+ = bezeugt; - = nicht bezeugt)

Interne Kopulaprädikation kann mit Possession (índraśatru-) und Kausativ (vrștídyu-) kombiniert werden, aber naturgemäss nicht mit einem S/A-Verb-Verhältnis in der externen Relation. Interne Modifikation kann ihrerseits zusammen mit Possession (áyaḩsthūna-) und mit einem S/A-VerbVerhältnis (raghuyáman-) verwendet werden, aber nicht mit dem externen Kausativverhältnis. Das interne Verb-Argument-Verhältnis ist weder mit Possession noch mit dem Kausativ kombinierbar, dafür exklusiv mit einem S/A-Verb-Verhältnis in der externen Relation (ánnakāma-). In der externen Relation ist nach der Definition hier das Kausativverhältnis exklusiv mit der Kopulaprädikation kombinierbar.

Die Berücksichtigung aller drei Relationen und insbesondere der internen und externen Relationen bietet eine geeignete Matrix für die Beschreibung von Possessivkomposita, verbalen Rektionskomposita und kausativen Komposita, die im wesentlichen durch die Existenz der externen Relation zwischen Z und $\mathrm{Y}(/ \mathrm{X})$ eine kohärente Gruppe bilden.

Was hier nicht näher untersucht wurde, ist die Frequenz, mit der die einzelnen Typen und die Kombinationen der Typen auftreten. Dies setzt umfassende Erhebungsarbeiten voraus, die in diesem Rahmen nicht geleistet werden konnten. Weiter wurde auch die Relation zwischen dem Gesamtkompositum und dem Bezugsnomen sowie die Funktionen, die diese Komposita in unterschiedlichen Ausprägungen in der infiniten Satzverknüpfung wahrnehmen können, nicht in die Diskussion miteinbezogen.

Das hier vorgeschlagene Gerüst mit seinen Kombinationen aus externer und interner Relation stellt gemäss der Zielsetzung ein Set an Interpretationsmöglichkeiten für trirelationale exozentrische Komposita zur Verfügung. Es ist in diesem Zusammenhang wichtig, noch einmal die Relevanz der Semantik 
der involvierten drei Begriffe, der Kontextgebundenheit und der Pragmatik bei der Interpretation der hier beschriebenen Syntagmen hervorzuheben: Die zutreffenden (oder möglichen) Interpretationen resultieren immer erst aus dem Zusammenspiel der drei involvierten Elemente und der Relationen, die sich im Kontext und aus den referentiellen Eigenschaften der Begriffe ergeben.

Danksagung: Wir danken den beiden anonymen Gutachtern für konstruktive Kritik und Verbesserungsvorschläge, Florian Sommer für Diskussion und Elisabeth Rieken für Kommentare zu einer früheren Version dieses Beitrags.

\section{Literatur}

Amano, Kyōko (2009). Maitrāyaṇi Samihitā I-II. Übersetzung der Prosapartien mit Kommentar zur Lexik und Syntax der älteren vedischen Prosa. Münchner Forschungen zur historischen Sprachwissenschaft 9. Bremen: Hempen.

Bauer, Laurie (2009). „Typology of compounds“. In: The Oxford Handbook of Compounding. Hrsg. von Rochelle Lieber \& Pavol Štekauer. Oxford Handbooks in Linguistics. Oxford: Oxford University Press, 343-356.

Brugmann, Karl (1906). Grundriss der vergleichenden Grammatik der indogermanischen Sprachen. Bd. 2: Lehre von den Wortformen und ihrem Gebrauch. 1. Allgemeines, Zusammensetzung (Komposita), Nominalstämme. 2. Aufl. Strassburg: Trübner.

Dunkel, George E. (1992). „Two old problems in Greek: $\pi \tau \dot{\lambda} \lambda \varepsilon \mu \circ \varsigma$ and $\tau \varepsilon \rho \psi i ́ \mu \beta \rho \circ \tau \circ \varsigma^{\prime \prime}$ In: Glotta 70, 197225 .

Hoffmann, Karl (1975-1992). Aufsätze zur Indoiranistik. 3 Bde. Wiesbaden: Reichert.

Jacobi, Hermann (1897). Kompositum und Nebensatz. Studien über die indogermanische Sprachentwicklung. Bonn: Cohen.

Jamison, Stephanie W. \& Joel P. Brereton (2014). The Rigveda. The Earliest Religious Poetry of India. 3 Bde. New York: Oxford University Press.

Janda, Michael (1999). „Evooí $\theta \omega \omega u^{\prime \prime}$ In: Compositiones Indogermanicae in memoriam Jochem Schindler. Hrsg. von Heiner Eichner \& Hans-Christian Luschützky. Praha: Enigma, 183-203.

Justi, Ferdinand (1861). Ueber die zusammensetzung der nomina in den indogermanischen sprachen. Göttingen: Dieterich.

Kastovsky, Dieter (2009). „Diachronic perspectives“. In: The Oxford Handbook of Compounding. Hrsg. von Rochelle Lieber \& Pavol Štekauer. Oxford Handbooks in Linguistics. Oxford: Oxford University Press, $323-340$.

Liddell, Henry G. u. a. (1996). A Greek-English Lexicon. 9. Aufl. Oxford: Clarendon Press. 
Lieber, Rochelle \& Pavol Štekauer (2009). „Introduction: status and definition of compounding“. In: The Oxford Handbook of Compounding. Hrsg. von Rochelle Lieber \& Pavol Štekauer. Oxford Handbooks in Linguistics. Oxford: Oxford University Press, 3-18.

Lindner, Thomas (2011-). Indogermanische Grammatik. Bd. 4.1: Komposition. Heidelberg: Winter.

Lühr, Rosemarie (2004). „Nominalkomposition im Altindischen und Altgriechischen“. In: Komplexe Wortstrukturen. Komposition, Inkorporation, Polysynthese. Hrsg. von Elke Nowak. Berlin: Institut für Sprache und Kommunikation, TU Berlin, 107-214.

Macdonell, Arthur A. (1910). Vedic Grammar. Strassburg: Trübner.

Nielsen Whitehead, Benedicte (2012). Pickpocket compounds from Latin to Romance. Proefschrift. Leiden: Leiden University. URN:NBN:NL:UI:26-1887/18706.

Ramharter, Hans (2012) . „Gebetene und ungebetene Gäste: Zur Etymologie des indoiran. 'Gast'-Wortes (ved. átit ${ }^{h}$-, av. asti-) und westidg. " $g^{h}$ óstis". In: Iranistische und indogermanische Beiträge in memoriam Jochem Schindler (1944-1994). Hrsg. von Velizar Sadovski \& David Stifter. Veröffentlichungen zur Iranistik 51. Österreichische Akademie der Wissenschaften.

Sadovski, Velizar (2000). „Die exozentrischen Zusammensetzungen mit Vorderglied Präverb/Präposition im Rgveda: Entheos-Komposita und präpositionale Rektionskomposita“. In: Indoiranisch, Iranisch und die Indogermanistik. Arbeitstagung der Indogermanischen Gesellschaft vom 2. bis 5. Oktober 1997 in Erlangen. Hrsg. von Bernhard Forssman \& Robert Plath. Wiesbaden: Reichert, 455-473.

- (2002). „Dvandva, tatpurușa and bahuvrihi. On the Vedic sources for the names of the compound types in Pāninin's Grammar“. In: Nominal Composition in Indo-European. Hrsg. von Torsten Meissner \& James Clackson. Transactions of the Philological Society 100.3, 351-402.

- (2004). „Komposita mit prädikativischem bzw. partitivischem Attributionsverhältnis der Glieder im Iranischen und Indischen“. In: Indo-European Word Formation. Proceedings of the Conference held at the University of Copenhagen, October $20^{\text {th }}-22^{\text {th }}$ 2000. Hrsg. von James Clackson \& Birgit A. Olsen. Copenhagen: Museum Tusculanum, 134-167.

Scalise, Sergio \& Antonietta Bisetto (2009). „The classification of compounds“. In: The Oxford Handbook of Compounding. Hrsg. von Rochelle Lieber \& Pavol Štekauer. Oxford Handbooks in Linguistics. Oxford: Oxford University Press, 34-53.

Scarlata, Salvatore (1999). Die Wurzelkomposita im Rg-Veda. Wiesbaden: Reichert.

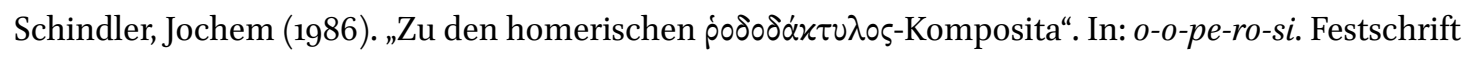
für Ernst Risch zum 75. Geburtstag. Hrsg. von Annemarie Etter. Berlin \& New York: de Gruyter, 393-401.

- (1997). „Zur internen Syntax der indogermanischen Nominalkomposita“. In: Berthold Delbrücky la sintaxis indoeuropea hoy. Hrsg. von Emilio Crespo \& José Luis García Ramón. Madrid \& Wiesbaden: Reichert, 537-540. 
Schroeder, Leopold (1874). Ueber die formelle Unterscheidung der Redetheile im Griechischen und Lateinischen mit besonderer Berücksichtigung der Nominalcomposita. Leipzig: in Komm. bei Köhler.

Wackernagel, Jacob (1905). Altindische Grammatik. Bd. 2, 1: Einleitung zur Wortlehre. Nominalkomposition. Göttingen: Vandenhoeck \& Ruprecht.

Whitney, William D. (1896). A Sanskrit grammar. Including both the classical language, and the older dialects, of Veda and Brahmana. Leipzig \& Boston: Breitkopf und Härtel \& Ginn. 